BULL. AUSTRAL. MATH. SOC.

VOL. 27 (1983), 339-345.

\title{
A CHARACTERIZATION OF REAL AND COMPLEX HILBERT SPACES \\ AMONG ALL NORMED SPACES
}

\author{
J. VUKMAN
}

Let $X$ be a real or complex normed space and $L(X)$ the algebra of all bounded linear operators on $X$. Suppose there exists a *-algebra $B(X) \subset L(X)$ which contains the identity operator $I$ and all bounded linear operators with finite-dimensional range. The main result is: if each operator $U \in B(X)$ with the property $U^{*} U=U U^{*}=I$ has norm one then $X$ is a Hilbert space.

In our terminology, an inner product is a real (complex) valued function $(\cdot, \cdot)$ defined for all pairs $x, y$ on a real (complex) vector space $X$ so that the conditions

(i) $\left(\alpha_{1} x_{1}+\alpha_{2} x_{2}, y\right)=\alpha_{1}\left(x_{1}, y\right)+\alpha_{2}\left(x_{2}, y\right)$,

(ii) $(y, x)=(x, y) \quad((y, x)=\overline{(x, y)}$ in the complex case $)$

are fulfilled. An inner product is said to be positive definite (negative definite $)$ if $(x, x)>0$ for all $x \in X, x \neq 0 \quad((x, x)<0$ for all $x \in X, x \neq 0)$.

Throughout this paper we denote by $L(X)$ the algebra of all bounded linear operators on a real or complex normed space $X$, and by $X^{*}$ the dual space of $X$. We shall write $x \otimes f$ for the bounded linear operator defined by $(x \otimes f) y=f(y) x$, where $f \in X^{*}$ is a fixed functional and $x \in X$ a fixed vector. We shall write $B(X)$ for any subalgebra of $L(X)$

Received 4 January 1983. 
which contains all bounded linear operators with finite-dimensional range. By involution we mean a linear (in the complex case a conjugate linear) mapping $A \longmapsto A^{*}$ on $L(X)$ or $B(X)$ such that $(A B)^{*}=B^{*} A^{*}$ and $A^{* *}=A$. Let a $B(X)$ equipped with an involution be given. An operator $U \in B(X)$ will be called unitary if $U^{*} U=U U^{*}=I, A \in B(X)$ will be called normal if $A^{*} A=A A^{*}$, and $H \in B(X)$ is hermitian if $H^{*}=H$.

Let $X$ be such a real or complex Banach soace that there exists an involution $A \mapsto A^{*}$ on $L(X)$ satisfying the condition $A^{*} A \neq 0$ for each nonzero $A \cdot \in L(X)$. According to the classical result of Kakutan $i$ and Mackey (see [2] and [3]) there exists a positive definite inner product on $X$ such that the corresponding norm is equivalent to the given norm on $X$, and that $A^{*}$ is the adjoint of $A$ relative to the inner product (see also [1]). Some results in the sense of the Kakutani-Mackey theorem can be found in our earlier paper [7]. The main purpose of this paper is to prove the result below which characterizes real or complex Hilbert spaces among all real or complex normed spaces in terms of involution on $B(X)$.

THEOREM 1. Let $X$ be a real or complex normed space. Suppose there exists an involution $A \mapsto A^{*}$ on some $B(X)$. If each unitary operator has norm one then there exists a positive definite inner product $(\cdot, \cdot)$ on $X$ such that the corresponding norm is equal to the given norm. For each $A \in B(X)$ the relation $(A x, y)=\left(x, A^{*} y\right)$ holds for all pairs $x, y \in X$.

REMARK. Since in the result above we did not require any completeness assumption, let us point out that there exists a counter-example which shows that the Kakutani-Mackey theorem cannot be proved without the assumption that $X$ is complete (see [2]).

Proof of Theorem 1. First we shall prove that for an arbitrary projector $P \in B(X)$ the relation $P^{*} P=0$ implies $P=0$. Suppose on the contrary that there exists $P \neq 0$ such that $P^{*} P=0$. Then a routine calculation shows that for any real number $t$ the operator $U_{t}$ defined by the relation

$$
U_{t}=I+(\exp t-1) P+(\exp (-t)-1) P^{*}-\frac{1}{2}(\exp t+\exp (-t)-2) P P^{*}
$$

is unitary. Let $e \in X$ be such that $P e=e,\|e\|=1$. Then $P^{*} P=0$ implies $P^{*} e=0$. Using this we obtain $U_{t} e=(\exp t) e$. Therefore 
$\left\|U_{t}\right\| \geq\left\|U_{t} e\right\|=\exp t$. This inequality is in contradiction with the requirement of the theorem that $\left\|U_{t}\right\|=1$. So the implication $P^{*} P=0 \Rightarrow P=0$ is proved.

Let $H \in B(X)$ be a one-dimensional hermitian operator. Let us denote by $L$ the left ideal $B(X) H$. Since $H$ is one-dimensional and hermitian, one can introduce an inner product into $L$ as follows:

$$
(A, B) H=B^{*} A, A, B \in L \text {. }
$$

It is easy to check that $(\cdot, \cdot)$ is indeed an inner product. The operator $H$ can be expressed in the form

$$
H=e \otimes f_{0}
$$

where $e \in X$ is a fixed nonzero vector, and $f_{0} \in X^{*}$ a fixed nontrivial functional. Obviously, the left ideal $L$ contains exactly those operators which can be written in the form $x \otimes f_{0}$, where $f_{0}$ is the functional from (1) and $x \in X$ an arbitrary vector. The isomorphism $x \mapsto x \otimes f_{0}$ allows us to introduce an inner product into $X$ as follows:

$$
(x, y)=\left(x \otimes f_{0}, y \otimes f_{0}\right), x, y \in X .
$$

Let us prove that

$$
(A x, y)=\left(x, A^{*} y\right)
$$

holds for each $A \in B(X)$ and all pairs $x, y \in X$. Using the relation $(A x) \otimes f_{0}=A\left(x \otimes f_{0}\right)$, we obtain

$(A x, y) H=\left((A x) \otimes f_{0}, y \otimes f_{0}\right) H$

$$
=\left(A\left(x \otimes f_{0}\right), y \otimes f_{0}\right) H=\left(y \otimes f_{0}\right) *_{A}\left(x \otimes f_{0}\right) .
$$

On the other hand

$$
\begin{aligned}
\left(x, A^{*} y\right) H=\left(x \otimes f_{0},\left(A^{*} y\right) \otimes f_{0}\right) H & =\left(x \otimes f_{0}, A^{*}\left(y \otimes f_{0}\right)\right) H \\
& =\left(A^{*}\left(y \otimes f_{0}\right)\right) *\left(x \otimes f_{0}\right)=\left(y \otimes f_{0}\right) * A\left(x \otimes f_{0}\right) .
\end{aligned}
$$

Now we intend to prove that for each $y \in X$ there corresponds a constant $C_{y}$ such that

$$
|(x, y)| \leq c_{y}\|x\|
$$


holds for all $x \in X$. Let $e \in X$ and $f_{0} \in X^{*}$ be from (1), and let $u \in X$ be such that $f_{0}(u)=1$. Then

$$
\begin{aligned}
|(x, y)|\|e\|=\|(x, y) H u\| & =\left\|\left(x \otimes f_{0}, y \otimes f_{0}\right) H u\right\| \\
& =\left\|\left(y \otimes f_{0}\right) *\left(x \otimes f_{0}\right) u\right\|=\left\|\left(y \otimes f_{0}\right) * x\right\| \leq\left\|\left(y \otimes f_{0}\right) *\right\|\|x\| .
\end{aligned}
$$

Therefore $|(x, y)| \leq\|e\|^{-1}\left\|\left(y \otimes f_{0}\right) *\right\|\|x\|$ which proves the relation (4).

The inequality (4) means that for each $y \in X$ the linear functional $f_{y}$ defined by $f_{y}(x)=(x, y)$ is continuous. Let us prove the converse. More precisely, we intend to show that each continuous linear functional $f \in X^{*}$ can be written in the form $f(x)=(x, y)$ for some fixed $y \in X$. Let therefore $f \in X^{*}$ be arbitrary, and let us choose fixed vectors $u$ and $v$ such that $(u, v)=1$. Then using the relation (3) we obtain $f(x)=((u \otimes f) x, v)=\left(x,(u \otimes f)^{*} v\right)$.

Let us prove that $(x, x)=0$ implies $x=0$. Suppose on the contrary that there exists a nonzero vector $u \in X$ such that $(u, u)=0$. Let $f \in X^{*}$ be such that $f(u)=1$. Then $P=u \otimes f \neq 0$. For all pairs $x, y \in X$ we have $\left(P^{*} P x, y\right)=(P x, P y)=(f(x) u, f(y) u)=0$. Therefore $P^{*} P=0$ which is in contradiction with $P \neq 0$ since we have proved that for all idempotents $P \in B(X)$ the implication $P^{*} P=0 \Rightarrow P=0$ is fulfilled.

From the implication $(x, x)=0 \Rightarrow x=0$ it follows easily that the inner product is positive or negative definite. We may assume that the inner product is positive definite, since in the case of a negative definite inner product we can introduce a positive definite inner product $(\cdot, \cdot)_{0}$ by $(x, y)_{0}=-(x, y)$.

Therefore there are two norms on $X$, the original one, and the norm induced by the inner product. We have to prove that there exists such an inner product on $X$ that the corresponding norm is equal to the given norm. It suffices to prove that the following implication

$$
\left(e_{1}, e_{1}\right)=\left(e_{2}, e_{2}\right)=1 \Rightarrow\left\|e_{1}\right\|=\left\|e_{2}\right\|
$$

holds for all $e_{1}, e_{2} \in X$. Let therefore $e_{1}, e_{2} \in X$ be such that 
$\left(e_{1}, e_{1}\right)=\left(e_{2}, e_{2}\right)=1$, and let us first assume that

$$
\left(e_{1}, e_{2}\right)=0
$$

Let us define $P_{1}$ and $P_{2}$ by the relations $P_{1} x=\left(x, e_{1}\right) e_{1}$, $P_{2} x=\left(x, e_{2}\right) e_{2}$. It is easy to see that the idempotents $P_{1}$ and $P_{2}$ are hermitian. From (6) we obtain $P_{1} P_{2}=P_{2} P_{1}=0$, whence it follows that the hermitian operator $P=P_{1}+P_{2}$ is also idempotent. Obviously, $e_{1}$ and $e_{2}$ are linearly independent. Denote by $X_{2}$ the two-dimensional subspace of $X$ determined by $e_{1}$ and $e_{2}$, and let a subspace $X_{c} \subset X$ be such that $X=X_{2} \oplus X_{c}$ is a decomposition of $X$ made by the projector $P$. Then each $x \in X$ can be uniquely expressed in the form $x=\lambda_{1} e_{1}+\lambda_{2} e_{2}+x_{c}, \quad x_{c} \in X_{c}$, which allows us to introduce a linear operator $U$ by the relation $U x=-\lambda_{2} e_{1}+\lambda_{1} e_{2}+x_{c}$. It is not difficult to see that $U \in B(X)$ and that $U$ is unitary. From the requirement of the theorem that each unitary operator has norm one it follows that $\|U x\|=\|x\|$. Therefore since $U e_{1}=e_{2}$ we have $\left\|e_{2}\right\|=\left\|U e_{1}\right\|=\left\|e_{1}\right\|$, which proves the implication (5) for the special case $\left(e_{1}, e_{2}\right)=0$. Let us prove the general case. Let therefore $e_{1}, e_{2} \in X$ be such vectors that $\left(e_{1}, e_{1}\right)=\left(e_{2}, e_{2}\right)=1$. There exists a nontrivial functional $f \in X^{*}$ such that $f\left(e_{1}\right)=f\left(e_{2}\right)=0$. Since we have proved that each continuous linear functional can be represented by the inner product, it follows that there exists a nonzero vector $e \in X$ such that $\left(e_{1}, e\right)=\left(e_{2}, e\right)=0$. We may assume that $(e, e)=1$. Hence $\left\|e_{1}\right\|=\|e\|=\left\|e_{2}\right\|$ which proves the implication (5) in its full generality.

It remains to show that $X$ equipped with the inner product is not only a pre-Hilbert but even a Hilbert space. This follows from the fact that a pre-Hilbert space in which the Riesz representation theorem holds is complete. The proof of the theorem is complete.

REMARK. Theorem 1 is proved in [8] using a different approach and the stronger assumption that $X$ is complete. 
COROLLARY 2. Let $X$ be a real or complex normed space. Suppose that there exists an involution $A \mapsto A^{*}$ on some $B(X)$ such that $\left\|A^{*} A\right\|=\|A\|^{2}$ holds for all normal operators $A \in B(X)$. In this case there exists a positive definite inner product $(\cdot, \cdot)$ on $X$ such that the corresponding norm is equal to the given norm. For each $A \in B(X)$ the relation $(A x, y)=\left(x, A^{*} y\right)$ is fulfilled for alz pairs $x, y \in X$.

REMARK. It should be mentioned that the complex version of Corollary 2 was first proved by Prijatelj [4] by a different method and stronger assumptions. He required that $X$ is complete, that the relation $\left\|A^{*} A\right\|=\|A\|^{2}$ is fulfilled for each $A \in L(X)$, and used methods from $B^{*}-$ algebra theory. Another improvement of Prijatelj's result can be found in [6].

\section{References}

[1] J. Bognár, "Star-algebras induced by non-degenerate inner products", Acta Sci. Math. 39 (1977), 15-26.

[2] Shizuo Kakutani and George W. Mackey, "Two characterizations of real Hilbert space", Ann. of Math. (2) 45 (1944), 50-58.

[3] Shizuo Kakutani and George W. Mackey, "Ring and lattice characterizations of complex Hilbert space", Bulz. Amer. Math. Soc. 52 (1946), 727-733.

[4] Niko Prijatelj, "Algebra characterization of Hilbert space", Publ. Math. (Ljubzjana) 1 (1964), 43-57.

[5] Charles E. Rickart, General theory of Banach algebras (Van Nostrand, Princeton, New Jersey, 1970).

[6] J. Vukman, "Characterization of Hilbert space", Glasnik Mat. 15 (35) $(1980), 301-303$.

[7] J. Vukman, "Involution on $L(X)$ ", Glasnik Mat. 17 (37) (1982), 65-72. 
[8] Joso Vukman, "A characterization of real and complex Hilbert space", Glasnik Mat. (to appear).

University of Maribor,

Veks,

Razlagova 14,

62000 Maribor,

Yugoslavia. 